\title{
Quantitative magnetic resonance imaging analysis correlates with surgical outcome of cervical spinal cord injury without radiologic evidence of trauma
}

\author{
L-Q Sun ${ }^{1}$, Y Shen ${ }^{1}$ and $\mathrm{Y}-\mathrm{M} \mathrm{Li}{ }^{2}$
}

Study design: Prospective study.

Objectives: To investigate whether pre- and post-operative changes of signal intensity (SI) and transverse area (TA) of the spinal cord on T2-weighted magnetic resonance imaging (MRI) reflect the surgical outcome in patients with spinal cord injury (SCI) without radiologic evidence of trauma (SCIWORET).

Setting: The Third Hospital of Hebei Medical University, Shijiazhuang, Hebei, China.

Methods: In 36 consecutive prospective patients, MRI was performed pre-operatively and 3 months post-operatively. The Japanese Orthopaedic Association (JOA) scale and the American Spinal Cord Injury Association (ASIA) motor score (AMS) were used to quantify neurologic status at admission and at least 12-month follow-up. Pre- and post-operative TA, range of signal intensity (RSI), grayscale of signal intensity (GSI) and prevertebral hyperintensities (PVHs) were measured using the image analysis software. Pre-operative status and post-operative recovery were assessed in relation to MRI parameters pre- and post-operatively using univariate and multivariate analysis.

Results: Pre-operative JOA and AMS score negatively correlates RSI, GSI and PVH. There was no significant correlation between pre-operative TA and pre-operative JOA and AMS. Recovery rate with JOA negatively correlates pre-operative RSI, post-operative RSI, pre-operative GSI, post-operative GSI and PVH. There was a significant negative correlation between recovery rate with AMS and pre-operative RSI, post-operative GSI and PVH. From these results of multivariate stepwise regression analysis, the predictors of surgical outcomes are pre-operative GSI and pre-operative RSI.

Conclusion: Quantitative MRI analysis may provide reliable information for the prediction of the initial neurological status and surgical outcome of patients with SCIWORET.

Spinal Cord (2014) 52, 541-546; doi:10.1038/sc.2014.60; published online 6 May 2014

\section{INTRODUCTION}

Spinal cord injury (SCI) without radiographic abnormalities was first described by Pang et al. ${ }^{1,2}$ and defined as a clinicoradiological entity that presents as acute traumatic myelopathy with normal radiographic and computed tomography findings. However, the term 'spinal cord injury without radiologic evidence of trauma (SCIWORET)' was recommended to be used for the adult population, to include the situation that plain radiographs and computed tomography scan do not show any evidence of trauma except for pre-existing cervical spondylosis. ${ }^{3-5}$ Surgical intervention is recommended by many authors under the circumstances of cervical cord compression on magnetic resonance imaging (MRI) responsible for neurological deficits in older patients with SCIWORET as reported outcomes with conservative management may have profound residual deficits, particularly in the hands, ${ }^{6-8}$ and minor trauma may induce symptomatic spinal cord compression unexpectedly due to a pre-existing pathology, despite the patient being asymptomatic before the trauma. How to predict neurological outcomes after surgery is of great importance for the management of the patients with SCIWORET.
There is no doubt that MRI is the best radiological modality for examination and evaluation of patients with SCIWORET. MRI can show not only the degree of spinal degeneration but also the intramedullary state of the spinal cord in detail. ${ }^{9}$ MRI is helpful in both the diagnosis and prognosis of SCIWORET because it can not only reliably reveal intrinsic injury to the spinal cord, clearly depicting lesion location, extent and severity, but also provide the best imaging evaluation of the intervertebral discs, the adjacent ligaments and soft tissues.

MRI-documented intramedullary cord changes are well-known changes in spinal cord lesions. These signal changes are considered to reflect various intramedullary lesions. ${ }^{10,11}$ To date, post-operative MRI has not yet been fully studied in relation to the correlation of morphology or signal change of cervical cord and longer term prognosis in patients with SCIWORET., ${ }^{5,14}$ In our study, we have focused on quantitative analysis of signal intensity (SI) and transverse area (TA) of the spinal cord on T2-weighted MRI pre- and postoperatively. The purpose of the present study was to investigate whether range of signal intensity (RSI), grayscale of signal intensity (GSI), TA of the spinal cord and prevertebral hyperintensities (PVHs) reflect the surgical outcome in patients with SCIWORET.

\footnotetext{
${ }^{1}$ Department of Spine Surgery, The Third Hospital of Hebei Medical University, Shijiazhuang, Hebei, China and ${ }^{2}$ Department of Spine Surgery, The Second Hospital of Tangshan, Tangshan, Hebei, China

Correspondence: Dr Y Shen, Department of Spine Surgery, The Third Hospital of Hebei Medical University, 139 Ziqiang Road, Shijiazhuang, Hebei 050051, China. E-mail: ts_sunlaiqing@163.com

Received 21 December 2013; revised 8 March 2014; accepted 28 March 2014; published online 6 May 2014
} 

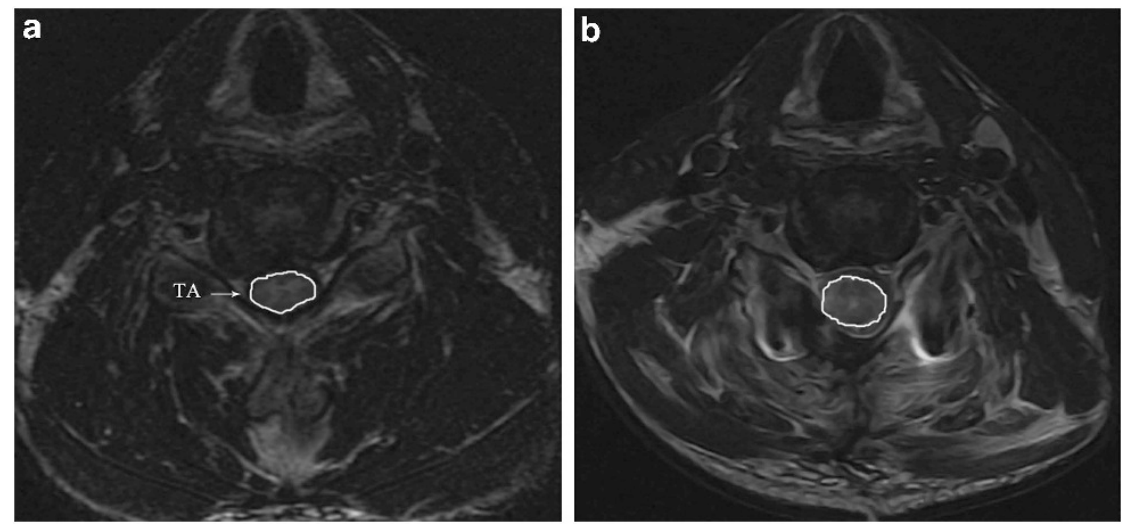

Figure 1 The TA of the spinal cord at the site of maximal compression was measured on the same axial T2WI pre-operatively (a) and post-operatively (b).

\section{MATERIALS AND METHODS}

Approval to conduct this study was obtained from our institutional review board, and informed consent was obtained from each patient. A total of 40 consecutive patients with SCIWORET who had undergone expansive laminoplasty in our institution from January 2011 to December 2011 prospectively enrolled and underwent MRI pre- and post-operatively in this study. After excluding 4 patients who were lost to follow-up, 36 cases (follow-up ratio: $90 \%$ ) were analyzed. In this study, SCIWORET was defined as: (1) the presence of neurologic deficit; (2) no signs of fracture or dislocation of the cervical spine on plain radiographs and computed tomography scans; and (3) the presence of cervical SCI on MRI. Patients with asymptomatic cervical cord compression, prior surgical intervention for CSM, infection, neoplastic disease, rheumatoid arthritis, ankylosing spondylitis, cerebrovascular accident and concomitant symptomatic lumbar spinal stenosis were excluded from the study.

\section{Procedures and outcome measures}

Data collection was performed by two of the authors (LQS and JMC) and externally monitored to ensure integrity and completeness. The criteria proposed by the Japanese Orthopedic Association (JOA) was used to assess neurologic status pre-operatively and post-operatively at least 12-month follow-up, and functional recovery rate was calculated by Hirabayashi's formula: ${ }^{15}$

Recovery rate with JOA

$=$ Post-operative JOA score $-\frac{\text { pre-operative JOA score }}{17(\text { full score })-\text { pre-operative JOA score } \times 100}$

The American Spinal Cord Injury Association (ASIA) scale was also used to evaluate the neurologic status and the ASIA motor score (AMS) is measured. Recovery rate with AMS was calculated by the Waters' formula: ${ }^{16}$

Recovery rate with AMS $=\frac{\text { Post-operative AMS }- \text { pre-operative AMS }}{100(\text { full score })-\text { pre-operative AMS } \times 100}$

\section{MRI interpretation}

In all the patients, MRI was performed just before surgery, then again 3 months afterward. All patients performed high-resolution MRI with a 1.5-Tesla imager (Signa, GE Medical Systems, Milwaukee, WI, USA). T1-weighted images (T1WI) and T2-weighted images (T2WI) of sagittal views of the cervical cord were obtained using a spin-echo sequence system for T1WI and a fast spin-echo sequence system for T2WI. Slice width was $3 \mathrm{~mm}$ and the acquisition matrix was $512 \times 512$. Sequence parameters were repetition time $1892 \mathrm{~ms}$ per echo time $10.1 \mathrm{~ms}$ for T1WI, and repetition time $2700 \mathrm{~ms}$ per echo time $123 \mathrm{~ms}$ for T2WI. Window width and level were set differently in each patient by the MR operators so that the optimal contrast between each tissue could be obtained.

MRI images were anonymous and were analyzed blindly by an experienced neuroradiologist using the image analysis software (Image J, free software, University of Toronto). The pre-and post-operative TA of the spinal cord at the site of maximal compression was measured on the same axial T2WI (Figure 1). The pre- and post-operative RSI was measured at the same sagittal view on T2-weighted MRI, while the pre- and post-operative GSI was calculated in MRT2WI sagittal views at the same spinal cord level and nearly same area PVHs were also measured at the sagittal view on T2-weighted MRI (Figure 2). Each was measured three times and the mean data were employed.

\section{Statistical analysis}

Descriptive statistics were used for all variables with distributions assessed for normality. The paired $t$-tests were conducted to compare pre-operative and post-operative changes in clinical outcomes and MRI parameters. Association was determined among variables using Spearman's rank correlation coefficient and multivariate linear regression models were computed using stepwise regression. Other statistical tests used included analysis of variance (ANOVA) and Student's $t$-test for continuous and categorical data, respectively. The Statistical Package for the Social Sciences (version 17.0 for Windows; SPSS Inc, Chicago, IL, USA) was used for statistical analysis. The difference was considered to be statistically significant at the $P<0.05$ level.

\section{RESULTS}

There were 27 men and 9 women. Mean ( \pm s.d.) age of this series was $52.2 \pm 10.9$ years (range, 23-78 years). Overall causes of injuries were trivial falls in 15 patients (41.7\%) and traffic injuries in 21 (58.3\%). All patients underwent expansive laminoplasty. In addition, three patients with pre-existing kyphosis or cervical instability underwent spinal instrumentation with fusion simultaneously. Pre-operative MRI was performed 1-4 days (mean 2.0) after the trauma. The interval between injury and surgery ranged from 2 to 7 days (mean 3.0). The mean follow-up period was 16 months (range, 13-19 months). The demographic and diagnostic characteristics of patients were listed in Table 1.

The mean JOA score rose from $7.97 \pm 4.67$ pre-operatively (range $1-15$ ) to $11.39 \pm 4.52$ (range $2-16$ ) post-operatively and the mean recovery rate with JOA was $43.87 \pm 19.36 \%$. The mean AMS rose from $57.75 \pm 31.14$ pre-operatively (range $8-96$ ) to $74.89 \pm 28.73$ (range 14-99) post-operatively and recovery rate with AMS was $50.44 \pm 28.47 \%$. There was a significant difference between preand post-operative JOA score $(t=-9.626 ; P<0.0001)$ and AMS $(t=-6.528 ; P<0.0001)$, showing statistically significant improvement at the clinical symptoms. There was no significant difference between sex groups when comparing recovery rate with JOA score $(t=-1.67 ; P=0.105)$ and motor recovery percentage $(t=-1.7$; $P=0.97)$. Summary of the clinical outcome and MRI parameters were listed in Table 2 . 

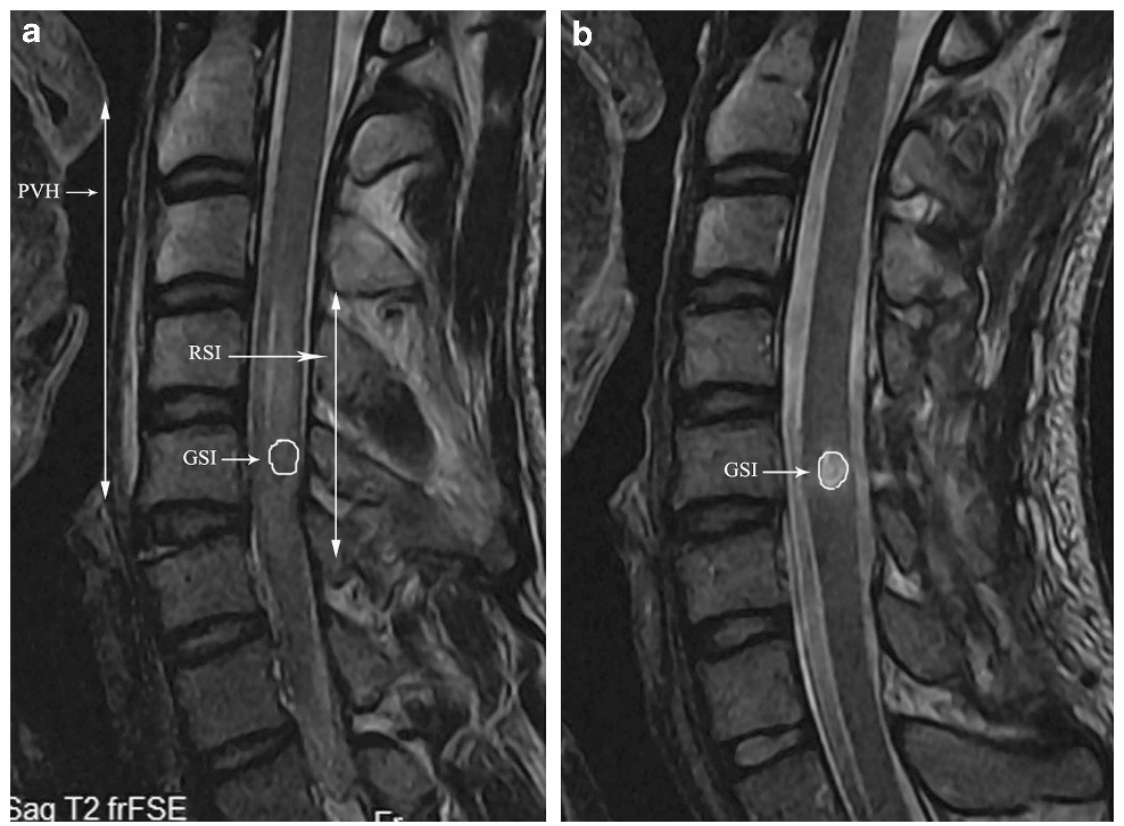

Figure 2 The RSI, the GSI and PVHs were calculated on sagittal T2WI at the same spinal cord level and nearly same area pre-operatively (a) and post-operatively (b).

All patients had adequate cord decompression as confirmed by MR images at 3 months after surgery. None of them required revision surgery for inadequate cord decompression. Post-operative complications related to cervical spine surgery occurred in 2 (5.6\%) of the 36 patients - 1 patient had cerebrospinal fluid leak which resolved at 8 days post-operatively and 1 patient had C5 palsy after laminectomy with instrumented fusion which resolved at 3 months postoperatively. No patients showed neurological deterioration at 12-month follow-up.

\section{Correlation of pre-operative JOA and AMS with patients' demographics and MRI}

Mean ( \pm s.d.) pre-operative RSI of this series was $24.25 \pm 10.31 \mathrm{~mm}$ (range $7.69-45.49 \mathrm{~mm}$ ). Mean ( \pm s.d.) pre-operative GSI of this series was $58.31 \pm 13.9$ (range 20.8-80.05). Mean ( \pm s.d.) PVH of this series was $36.66 \pm 25.93 \mathrm{~mm}$ (range $0-81.82 \mathrm{~mm}$ ). Pre-operative JOA score negatively correlates RSI $(r=-0.867, P<0.0001)$, GSI $(r=-0.533, P<0.001)$ and PVH $(r=-0.733, P<0.001)$. There was a significant negative correlation between pre-operative AMS with RSI $(r=-0.847, P<0.0001)$, GSI $(r=-0.541, P<0.001)$ and PVH $(r=-0.457, P=0.005)$.

Mean $\left( \pm\right.$ s.d.) pre-operative TA of this series was $73.3 \pm 12.83 \mathrm{~mm}^{2}$ (range 54.56-105 $\mathrm{mm}^{2}$ ). There was no significant correlation between pre-operative TA and pre-operative JOA and AMS. Sex and age were not associated with pre-operative JOA and AMS.

The multiple regression equations by multivariate stepwise regression analysis are as follows:

$$
\begin{aligned}
\text { Pre-operative JOA } & =20.78-0.323(\text { pre-op RSI }) \\
& -0.039 \times(\mathrm{PVH})-0.06 \times(\text { pre-op GSI })\left(\text { adjusted } R^{2}\right. \\
& =0.802, P<0.0001) .
\end{aligned}
$$

Pre-operative AMS $=142-2.366 \times(\mathrm{RSI})-0.461 \times(\mathrm{GSI})$

(adjusted $\left.R^{2}=0.764, P<0.0001\right)$.
Correlation of post-operative outcomes with patients' demographics and MRI

Mean ( \pm s.d.) post-operative RSI of this series was $12.64 \pm 7.23 \mathrm{~mm}$ (range 1-31.2 mm). There was a significant difference between preoperative and post-operative RSI in this series $(t=7.817 ; P<0.001)$. Mean ( \pm s.d.) post-operative GSI of this series was $64.14 \pm 20.31$ (range 35.58-115.75). There was no significant difference between pre-operative and post-operative GSI in this series $(t=-1.976$; $P=0.056)$. Mean ( \pm s.d.) post-operative TA of this series was $84.61 \pm 13.82 \mathrm{~mm}^{2}$ (range $64.8-115.79 \mathrm{~mm}^{2}$ ). There was a significant difference between pre-operative and post-operative TA in this series $(t=-6.234 ; P<0.001)$.

Recovery rate with JOA negatively correlates pre-operative RSI ( $r=-0.485, P=0.003)$, post-operative RSI $(r=-0.331, P=0.049)$, pre-operative GSI $(r=-0.494, \quad P=0.002)$, post-operative GSI $(r=-0.333, P=0.047)$ and PVH $(r=-0.460, P=0.005)$. There was a significant negative correlation between recovery rate with AMS and pre-operative RSI $(r=-0.535, P=0.01)$, post-operative GSI $(r=-0.502, P=0.002)$ and PVH $(r=-0.374, P=0.025)$. A trend toward a higher recovery rate with AMS was noted in patients with a lower post-operative RSI $(r=-0.326 ; P=0.053)$ and preoperative GSI $(r=-0.319 ; P=0.058)$, but this was not statistically significant. TA, age and sex were not associated with recovery rate.

The multiple regression equations by multivariate stepwise regression analysis are as follows:

$$
\begin{gathered}
\text { Recovery rate with JOA }(\%)=54.72+1.919 \times(\text { pre-operative JOA }) \\
-0.448 \times(\text { pre-operative GSI })\left(\text { adjusted } R^{2}=0.416, P<0.0001\right)
\end{gathered}
$$

Recovery rate with AMS $(\%)=85.44-1.443 \times($ pre-operative $\mathrm{RSI})$

$$
\text { (adjusted } \left.R^{2}=0.252, P<0.001\right) \text {. }
$$

\section{Correlation of TA with GSI, RSI and PVH}

Pre-operatively, TA has no correlation with RSI $(r=0.165, P=0.337)$, GSI $(r=0.086, P=0.616)$ and PVH $(r=-0.289, \quad P=0.087)$. 
Table 1 The demographic and diagnostic characteristics of patients with SCIWORET in this series

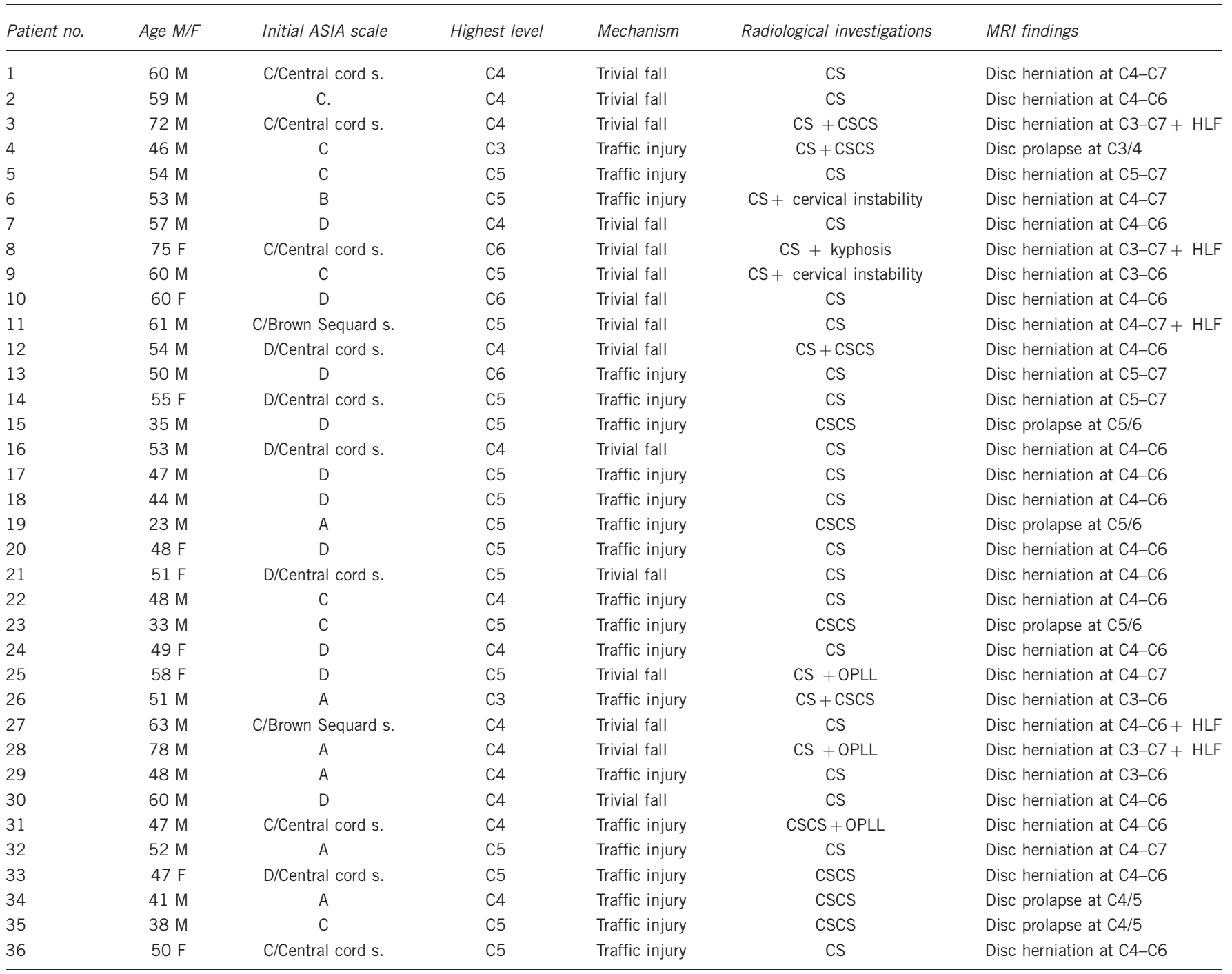

Abbreviations: CS, cervical spondylosis; CSCS, congenital spinal canal stenosis; HLF, hypertrophy of the ligamentum flava; OPLL, ossification of the posterior longitudinal ligament.

There was also no correlation of TA with RSI $(r=0.104, P=0.545)$ and GSI $(r=0.006, P=0.973)$ post-operatively.

All the correlation of MRI parameters with clinical outcomes were listed in Table 3.

\section{DISCUSSION}

Our study systematically assessed the variability between preand post-operative findings in evaluating the surgical outcome of cervical SCIWORET using quantitative MRI analysis. The present study suggests: (1) SI changes in the spinal cord on pre-operative MRI in patients with SCIWORET can reflect initial injury in the spinal cord, and to be indicative of the prognosis; (2) SI changes in the spinal cord on MRI performed 3 months after SCIWORET surgery correlate with longer term prognosis in SCIWORET patients, but is less reliable than pre-operative MRI in prediction of surgical outcome; (3) the degree of spinal cord compression, in the form of TA of the spinal cord, and age have no correlation with initial injury of spinal cord and surgical outcome.

\section{Clinical significance of SI of the spinal cord on MRI}

MRI has revolutionized the diagnosis of SCI, clearly depicting location, range and severity of different lesions. SI changes in the spinal cord on MRI in SCIWORET patients are thought to reflect pathologic changes in the spinal cord including hemorrhage, contusion or edema, and to be indicative of the prognosis. ${ }^{5,9,12,14}$ There were researches demonstrating in animal models that the extent of signal change shown on MRI is closely related to the severity of injury. ${ }^{17}$ With development in MRI techniques and software, it has now become possible to make quantitative analysis of RSI in a more comprehensive method. ${ }^{14}$ However, quantitative analysis of GSI, potentially valuable for the prediction of the outcome of surgical intervention for SCIWORET, has not yet been fully elucidated. Taking into account that MRI images capture various properties of biological architecture of spinal cord, the implementation of quantitative GSI analysis can provide quantitative metrics, relevant to the internal architecture and pathologic status of spinal cord. ${ }^{18}$ Statistical analysis demonstrated the existence of negative correlations of pre-operative GSI with pre-operative JOA or AMS score and recovery rate. Data from our study also support the fact that post-operative GSI 
Table 2 Summary of the clinical outcome and MRI parameters of this series

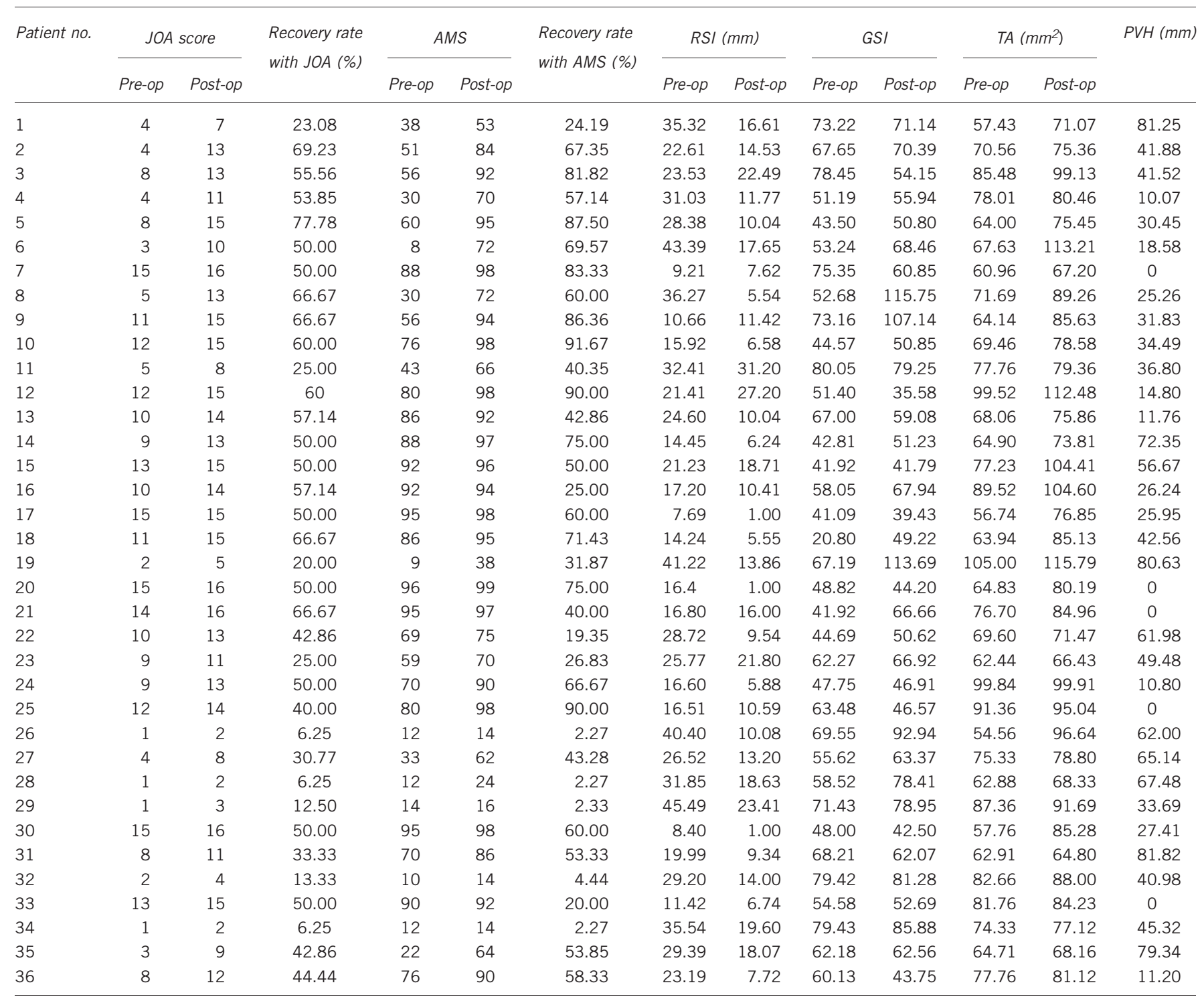

Abbreviations: AMS, American Spinal Cord Injury Association motor score; GSI, grayscale of signal intensity; JOA, Japanese Orthopaedic Association; MRI, magnetic resonance imaging; $\mathrm{PVH}$, prevertebral hyperintensity; RSI, range of signal intensity; TA, transverse area.

Table 3 Correlation of MRI parameters with clinical outcome

\begin{tabular}{|c|c|c|c|c|}
\hline & Pre-op JOA & Pre-op AMS & $R R$ with $J O A$ & $R R$ with $A M S$ \\
\hline \multicolumn{5}{|l|}{$R S I$} \\
\hline Pre-op RSI & $r=-0.867, P<0.0001$ & $r=-0.847, P<0.0001$ & $r=-0.485, P=0.003$ & $r=-0.535, P=0.01$ \\
\hline \multicolumn{5}{|l|}{ GSI } \\
\hline Pre-op GSI & $r=-0.533, P<0.001$ & $r=-0.541, P<0.001$ & $r=-0.494, P=0.002$ & $r=-0.319 ; P=0.058$ \\
\hline \multicolumn{5}{|l|}{$T A$} \\
\hline Pre-op TA & $r=-0.106, P=0.540$ & $r=-0.094, P=0.584$ & $r=0.40, P=0.817$ & $r=-0.013, P=0.939$ \\
\hline Post-op TA & & & $r=0.135 P=0.431$ & $r=0.096, P=0.587$ \\
\hline PVH & $r=-0.733, P<0.001$ & $r=-0.457, P=0.005$ & $r=-0.460, P=0.005$ & $r=-0.374, P=0.025$ \\
\hline
\end{tabular}

Abbreviations: AMS, American Spinal Cord Injury Association motor score; GSI, grayscale of signal intensity; JOA, Japanese Orthopaedic Association; MRI, magnetic resonance imaging; $\mathrm{PVH}$, prevertebral hyperintensity; RR, recovery rate; RSI, range of signal intensity; TA, transverse area. 
negatively correlates with recovery rate with JOA or AMS. All these results may be considered as indication of the fact that GSI, in terms of the internal architecture of spinal cord, closely correlates with initial injury of spinal cord and surgical outcome. Furthermore, our results reinforce previous researches that RSI in spinal cord and PVHs negatively correlates with initial injury of spinal cord and surgical outcome. $^{14}$

\section{Correlation of clinical outcomes with spinal cord compression}

There were researches suggest: axial sections based on T2-weighted MRI parameters were more reliable in assessing the degree of cervical spinal cord compression than T1-weighted sequences, and TA was reliable and versatile in assessing spinal cord compression. ${ }^{19}$ Data from our study support the fact that there was no correlation of TA with pre-operative JOA or AMS score and surgical outcome, suggesting that the extent of spinal cord compression has less of a predictive value in evaluating patients' neurological status and prognosis in the patients with SCIWORET. This is in accordance with previous research of ossification of posterior longitudinal ligament patients with traumatic cervical SCI. ${ }^{20}$ Trying to correlate TA with SI on MRI, one would expect TA to be larger in patients with lower RSI or GSI on MRI. However, contrary to expectation, there was no existence of statistically significant correlation between TA and RSI or GSI pre- and post-operatively. This study clearly demonstrated that RSI and GSI were significantly related to initial neurological status. Taken together, these findings indicate that the neurological status in the patients with SCIWORET mainly depends on the severity of initial injuries that occur at the time of trauma, and that SI of spinal cord and PVHs that can reflect the severity of initial injuries to spinal cord, rather than cervical canal stenosis, is important for determining the patients' prognosis.

\section{Predictors of surgical outcomes}

The current results showed no significant correlations of recovery rate with AMS and post-operative RSI or pre-operative GSI. However, there was a tendency toward negative correlation. These may result from the small sample capacity or a bias in patient selection. From these results of multivariate stepwise regression analysis, it can be concluded that the predictors of surgical outcomes are preoperative GSI and pre-operative RSI. Therefore, the current authors could possibly conjecture that pre-operative MRI is more reliable than MRI performed 3 months after surgery in prediction of surgical outcome.

Our study is limited by the low number of subjects and potential selection bias, as the result of infrequent condition of SCIWORET. All the patients with SCIWORET who had undergone expansive laminoplasty in our institution in 2011 were enrolled in this prospective study, so the selection bias may be avoided to some extent.

\section{DATA ARCHIVING}

There were no data to deposit.

\section{CONFLICT OF INTEREST}

The authors declare no conflict of interest.

1 Pang D, Pollack IF. Spinal cord injury without radiographic abnormality in children-the SCIWORET syndrome. J Trauma 1989; 29: 654-664.

2 Pang D, Wilberger JE Jr.. Spinal cord injury without radiographic abnormalities in children. J Neurosurg 1982; 57: 114-129.

3 Saruhashi Y, Hukuda S, Katsuura A, Asajima S, Omura K. Clinical outcomes of cervical spinal cord injuries without radiographic evidence of trauma. Spinal Cord 1998; 36: 567-573.

4 Hayashi K, Yone K, Ito H, Yanase M, Sakou T. MRI findings in patients with a cervical spinal cord injury who do not show radiographic evidence of a fracture or dislocation. Paraplegia 1995; 33: 212-215.

5 Kasimatis GB, Panagiotopoulos E, Megas P, Matzaroglou C, Gliatis J, Tyllianakis M et al. The adult spinal cord injury without radiographic abnormalities syndrome: magnetic resonance imaging and clinical findings in adults with spinal cord injuries having normal radiographs and computed tomography studies. J Trauma 2008; 65: 86-93.

6 Harrop JS, Sharan A, Ratliff J. Central cord injury: pathophysiology, management, and outcomes. Spine J 2006; 6 (S), 198-206.

7 Fehlings MG, Perrin RG. The role and timing of early decompression for cervica spinal cord injury: update with a review of recent clinical evidence. Injury 2005; 36: 13-26.

8 Yamazaki T, Yanaka K, Fujita K, Kamezaki T, Uemura K, Nose T. Traumatic centra cord syndrome: analysis of factors affecting the outcome. Surg Neurol 2005; 63 95-100.

9 Ohshio I, Hatayama A, Kaneda K, Takahara M, Nagashima K. Correlation between histopathologic features and magnetic resonance images of spinal cord lesions. Spine 1993; 18: 1140-1149.

10 Looby S, Flanders A. MRI of spinal cord injury. Cont Diagn Radiol 2012; 35: 1-6.

11 Do-Dai DD, Brooks MK, Goldkamp A, Erbay S, Bhadelia RA. Magnetic resonance imaging of intramedullary spinal cord lesions: a pictorial review. Curr Probl Diagn Radiol 2010; 39: 160-185.

12 Tewari MK, Gifti DS, Singh P, Khosla VK, Mathuriya SN, Gupta SK et al. Diagnosis and prognostication of adult spinal cord injury without radiographic abnormality using magnetic resonance imaging: analysis of 40 patients. Surg Neurol 2005; 63: 9 .

13 Hohl JB, Lee JY, Horton JA, Rihn JA. A novel classification system for traumatic central cord syndrome: the Central Cord Injury Scale (CCIS). Spine 2010; 35: 238-243.

14 Machino M, Yukawa Y, Ito K, Nakashima H, Kanbara S, Morita D et al. Can magnetic resonance imaging reflect the prognosis in patients of cervical spinal cord injury without radiographic abnormality? Spine 2011; 36: 1568-1572.

15 Hirabayashi K, Miyakawa J, Satomi K, Maruyama T, Wakano K. Operative results and post-operative progression of ossification among patients with ossification of cervical posterior longitudinal ligaments. Spine 1981; 6: 64.

16 Waters RL, Adkins RH, Sie IH, Yakura JS. Motor recovery following spinal cord injury associated with cervical spondylosis: a collaborative study. Spinal Cord 1996; 34 $711-715$.

17 Fujii H, Yone K, Sakou I. Magnetic resonance imaging study of experimental acute spinal cord injury. Spine 1993; 18: 2030-2034.

18 Lerski RA, Straughan K, Schad LR, Boyce D, Blüml S, Zuna I. MR image texture analysis-an approach to tissue characterization. Magn Reson Imaging 1993; 11: 873-887.

19 Karpova A, Arun R, Davis AM, Kulkarni AV, Mikulis DJ, Sooyong C et al. Reliability of quantitative Magnetic Resonance Imaging methods in the assessment of spinal canal stenosis and cord compression in cervical myelopathy. Spine 2013; 38: 245-252.

20 Okada S, Maeda T, Ohkawa Y, Harimaya K, Saiwai H, Kumamaru H et al. Does ossification of the posterior longitudinal ligament affect the neurological outcomes after traumatic cervical cord injury? Spine 2009; 34: 1148-1152. 